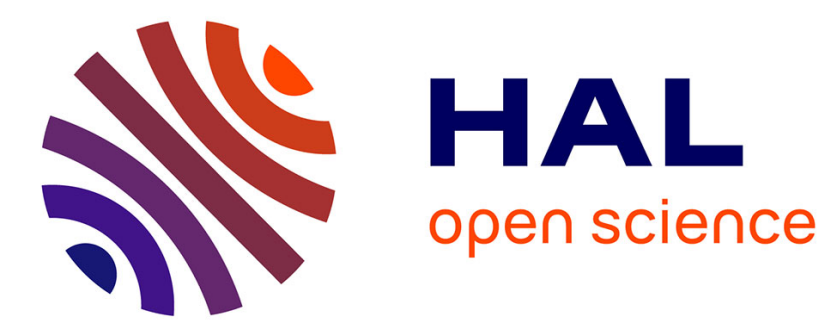

\title{
Resolving the role of magnetic circular dichroism in multishot helicity-dependent all-optical switching
}

\author{
Y. Quessab, M Deb, J. Gorchon, M. Hehn, G. Malinowski, S. Mangin
}

\section{To cite this version:}

Y. Quessab, M Deb, J. Gorchon, M. Hehn, G. Malinowski, et al.. Resolving the role of magnetic circular dichroism in multishot helicity-dependent all-optical switching. Physical Review B: Condensed Matter and Materials Physics (1998-2015), 2019, 10.1103/PhysRevB.100.024425 . hal-02184555

\section{HAL Id: hal-02184555 \\ https://hal.univ-lorraine.fr/hal-02184555}

Submitted on 16 Jul 2019

HAL is a multi-disciplinary open access archive for the deposit and dissemination of scientific research documents, whether they are published or not. The documents may come from teaching and research institutions in France or abroad, or from public or private research centers.
L'archive ouverte pluridisciplinaire HAL, est destinée au dépôt et à la diffusion de documents scientifiques de niveau recherche, publiés ou non, émanant des établissements d'enseignement et de recherche français ou étrangers, des laboratoires publics ou privés. 


\title{
Resolving the role of magnetic circular dichroism in multishot helicity-dependent all-optical switching
}

\author{
Y. Quessab, ${ }^{1,2, *}$ M. Deb, ${ }^{1,3}$ J. Gorchon, ${ }^{1}$ M. Hehn, ${ }^{1}$ G. Malinowski, ${ }^{1}{ }^{\dagger}$ and S. Mangin ${ }^{1}$ \\ ${ }^{1}$ Institut Jean-Lamour, CNRS UMR 7198, Université de Lorraine, 54011 Nancy, France \\ ${ }^{2}$ Center for Quantum Phenomena, Department of Physics, New York University, New York, 10003, USA \\ ${ }^{3}$ Institut für Physik und Astronomie, Universität Potsdam, 14476 Potsdam, Germany
}

(Dated: June 16, 2019)

\begin{abstract}
By conducting helicity-dependent ultrafast magnetization dynamics in a CoTb ferrimagnetic alloy, we are able to quantitatively determine the magnetic circular dichroism (MCD) and resolve its role in the helicity-dependent all-optical switching (AOS). Unequivocal interpretation of the sign of the dichroism is provided by performing AOS and femtosecond laser-induced domain wall motion experiments. We demonstrate that AOS occurs when the magnetization is initially in the most absorbent state according to the light helicity. Moreover, we evidence that the MCD creates a thermal gradient that drives a domain wall towards hotter regions. Our experimental results are in agreement with the purely thermal models of AOS.
\end{abstract}

The ultrafast optical control of the magnetic order rapidly emerged as a promising approach in ultrafast magnetism. Yet, a decade after the discovery of complete and deterministic magnetization switching induced by femtosecond circularly polarized laser pulses [1], many fundamental questions remained unanswered. In particular, the issue of the relative contribution of pure thermal or non-thermal effects induced by the laser pulses is crucial for the understanding of the magnetization reversal mechanism. Indeed, the interaction between light and matter may involve transfer of energy which results in an ultrafast heating of the electronic system [2-5]. Additionally, for circular polarization, transfer of light angular momentum and the emergence of an effective magnetic field via the inverse Faraday effect (IFE) may occur [6-9].

The ability to reverse the magnetization with ultrashort polarized laser pulses, a phenomenon known as alloptical switching (AOS), was evidenced in a broad variety of ferri- and ferro-magnetic materials $[1,10,11]$. In GdFeCo alloys, AOS can be single-pulse and helicityindependent $[3,4,12]$. On the other hand, in ferrimagnetic $\mathrm{CoTb}$ and ferromagnetic thin films such as $\mathrm{Co} / \mathrm{Pt}$ multiplayers, AOS is found to be helicity-dependent $[10,11,13,14]$. The latter was also demonstrated to be a multishot and cumulative process $[15-17]$ which involves domain nucleation and helicity-dependent domain wall propagation [18].

Several theoretical models have been developed for multi-pulse all-optical helicity-dependent switching (AOHDS) without reaching a consensus about the origin of the driving force $[16,19-22]$. To explain the role of the photon helicity, the IFE was considered among non-thermal optomagnetic effects. Following the laserinduced thermal demagnetization, the effective field generated by circularly-polarized laser pulses would switch the magnetization according to the light handedness $[9,19,20]$. This magnetization reversal mechanism is mostly non-thermal. Yet, a precise characterization of the amplitude and lifetime of this optomagnetic field re- mains elusive in absorbing metallic media $[9,19]$.

In contrast, it has been argued that AO-HDS could originate from a purely heating mechanism [16, 21, 22]. In this case, the asymmetry would arise from the difference in light absorption between left- and right- circular polarization due to the magnetic circular dichroism (MCD). The multishot helicity-dependence of AOS in ferromagnets was theoretically reproduced with a purely thermal mechanism based on the MCD [16, 21]. In this process, the laser initially heats the system close to the Curie temperature. As a result of the MCD, some magnetic domains would be hotter and will experience stochastic switching, while domains of opposite magnetization direction would remain cooler and stable [16, 21]. Moreover, it was suggested that the temperature gradient arising from the MCD across a domain wall could explain the helicity-dependent domain wall motion reported in $\mathrm{Co} / \mathrm{Pt}$ multilayers [18].

In all the thermal models of multishot AO-HDS [16, $21,22]$, it was assumed that the final state is the least absorbent. This means that switching occurs if the magnetization is initially in the higher absorption direction according to the light helicity. Hence, it comes that the sign of the MCD governs the helicity-dependence of AOS and the direction of the temperature gradient across a domain wall. Nevertheless, no experimental proof has been provided so far to corroborate the thermal models of AOS. Therefore, it appears legitimate to experimentally investigate the helicity dependence of light absorption in the magnetic thin films that exhibit multishot AO-HDS. A first attempt to reveal the MCD effects in $(\mathrm{Co} / \mathrm{Pt})$ multilayers upon laser pulse irradiation lacked of a direct comparison between the absorption states and the effect of the light helicity on the magnetization direction [23]. Besides, it is necessary to explore the effect of MCD on a domain wall as it was proved that domain wall motion plays an important role in the AOS mechanism [18].

In this letter, we aim to experimentally resolve the relevance of a purely thermal mechanism based on the 
MCD. Ultrafast magnetization dynamics and magnetooptical Kerr ellipticity measurements allow us to accurately determine the sign of the MCD. Unequivocal interpretation of the role of the MCD in the mechanism of AOS is provided by direct comparison to AO-HDS and helicity-dependent domain wall motion experiments.

To access the MCD, one can perform static absorption measurements. Yet, due to the small difference of absorption, it can be challenging to obtain the necessary signal to noise ratio for such measurements. Another possibility is to use ellipsometry to determine the complex refractive indices of both circular polarizations. However, in multilayers, the fitting procedures to extract the indices of refraction can quickly become complicated. For these reasons, and in order to access the MCD in-situ (in our AOS experimental setup) and avoid possible sign confusions due to changes of setup or configuration, we decided to measure the ultrafast magnetic dynamics of our samples under excitation by circularly polarized pulses [23].

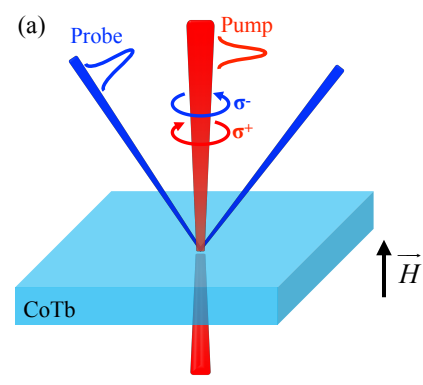

(b)

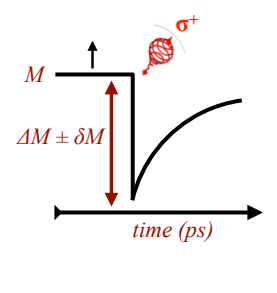

FIG. 1. Schematic representation (a) of the TR-MOKE configuration and (b) of the helicity-dependent magnetization dynamics.

The investigated sample is a ferrimagnetic alloy grown by DC magnetron sputtering: glass $/ \mathrm{Ta}(2) / \mathrm{Co}_{70} \mathrm{~Tb}_{30}(20) / \mathrm{Pt}(2)$ (thickness in $\mathrm{nm}$ ). The Ta seed layer is used for good adherence on the glass substrate and good texture to provide perpendicular magnetic anisotropy. The Pt capping layer prevents sample oxidation. Magnetometry measurement show that the CoTb ferrimagnetic alloy is Tb-dominant and with a coercive field of about $300 \mathrm{mT}$. Moreover, previous studies reported that CoTb alloys exhibit AO-HDS $[13,15,24]$. The ultrafast magnetization dynamics upon laser excitation was investigated via time-resolved magneto-optical Kerr effect (TR-MOKE) measurements using a pump-probe technique as sketched in Fig. 1(a). The pump and probe beams are generated by a 35 -fs pulsed laser with a repetition rate of $5 \mathrm{kHz}$. The pump wavelength is set to $800 \mathrm{~nm}$ while the frequency of the probe is doubled to $400 \mathrm{~nm}$. The pump beam is at normal incidence on the sample surface and the probe has a small incident angle of less than $5^{\circ}$. They both are focused onto the sample with a spot size of the pump and probe beams of about $280 \mu \mathrm{m}$ and $90 \mu \mathrm{m}$, respectively.
The laser-induced change of the Kerr rotation is detected with synchronized balanced photodiodes as a function of the time-delay between the pump and the probe. To explore the helicity dependence of the magnetization dynamics, the pump polarization was varied from left$\left(\sigma^{+}\right)$to right- $\left(\sigma^{-}\right)$circular polarization, on the other hand the probe was kept linearly-polarized.

Since the ultrafast magnetization dynamics in metallic systems depends on the amount of absorbed light [2, 25], it is therefore possible to extract the MCD from helicitydependent ultrafast magnetization dynamics. Indeed, in the linear regime, we can assume that the magnetization change is proportional to the absorbed laser fluence [26]. In Eq. 1, we define the magnetization change $M$ induced by a single laser pulse by a system of four equations depending on the light polarization and initial magnetization saturation direction as schematically illustrated in Fig. 1(b) (as explained in supplemental materials [27]). Typical time-resolved MOKE experiments require two measurements in order to disentangle the magnetic and non-magnetic contributions (e.g. reflectivity). Since MOKE is odd with the magnetization, the difference of two oppositely magnetized TR-MOKE traces will give a trace proportional to the magnetization, eliminating any effects that are even with the magnetization such as the reflectivity. Yet, for a circularly polarized pump, the probe's reflectivity will depend on the sign of $M$ due to the different amounts of heating caused by the MCD of the pump. As a result, it is necessary to measure the magnetization dynamics for all four combinations of light polarization and initial magnetization saturation direction $\left(\sigma^{-}, \uparrow\right),\left(\sigma^{-}, \downarrow\right),\left(\sigma^{+}, \uparrow\right)$ and $\left(\sigma^{+}, \downarrow\right)$. We note that this remains true for any time-resolved MOKE experiment where the pump beam has a non-negligible amount of helicity.

$$
\begin{aligned}
& M^{\left(\sigma^{-}, \uparrow\right)}=(\Delta R+\delta R)+(\Delta M+\delta M) \\
& M^{\left(\sigma^{-}, \downarrow\right)}=(\Delta R-\delta R)-(\Delta M-\delta M) \\
& M^{\left(\sigma^{+}, \uparrow\right)}=(\Delta R-\delta R)+(\Delta M-\delta M) \\
& M^{\left(\sigma^{+}, \downarrow\right)}=(\Delta R+\delta R)-(\Delta M+\delta M)
\end{aligned}
$$

$\Delta M$ refers to the helicity-independent magnetization change. $\delta M$ indicates the helicity-dependent component of the magnetization change. By reversing the light helicity, the demagnetization rate changes by $\pm \delta M$ which is directly proportional to the $M C D$. We define the measured change in reflectivity as an averaged (helicityindependent) reflectivity $\Delta R \pm \delta R$ related to its helicity dependence. By taking linear combinations of the four measurements as represented in Eq. 1, we can quantitatively determine $\delta M$, thus the $M C D$, and eliminate the helicity-independent and -dependent non-magnetic contributions $\Delta R$ and $\delta R$ (see. supplemental materials [27]). The convention used in Eq. 1 yields $\delta M$ proportional to 
$\left[A\left(\sigma^{-}, \uparrow\right)-A\left(\sigma^{-}, \downarrow\right)\right]$ (equivalently to $\left[A\left(\sigma^{+}, \downarrow\right)-A\left(\sigma^{+}\right.\right.$, $\uparrow)]$ ) with $A$ corresponding to the light absorption.

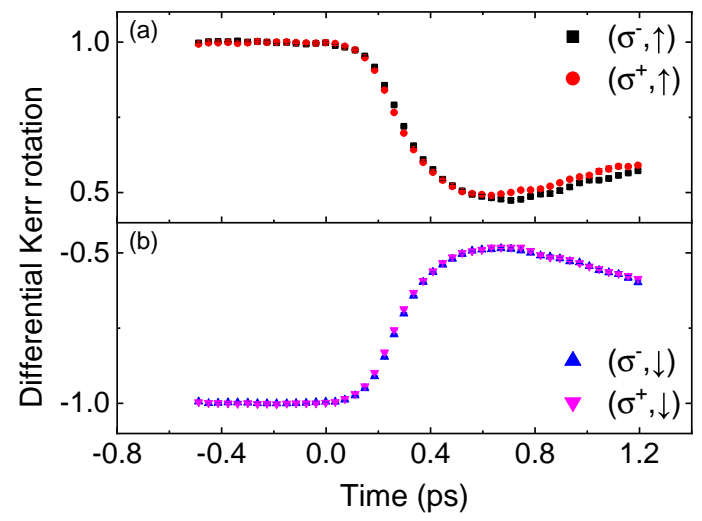

FIG. 2. Time-resolved magneto-optical Kerr effect measurements after a single left- $\left(\sigma^{+}\right)$or right- $\left(\sigma^{-}\right)$circularly polarized laser pulse for a magnetization pointing (a) up or (b) down.

In Figs. 2(a) and 2(b) are displayed the TR-MOKE results following a single laser pulse excitation in the CoTb alloy obtained for different combinations of light helicity and magnetization direction. Each ultrafast magnetization dynamics measurement was repeated 10 times and obtained with a temporal resolution of $35 \mathrm{fs}$. The error bars represent the standard error. The laser fluence was set to $4.87 \mathrm{~mJ} \mathrm{~cm}^{-2}$ with a magnetic field of 700 $\mathrm{mT}$ to re-set the magnetization. Due to the difference of absorption resulting from the MCD, for one saturation direction, one circular polarization will demagnetize more. By symmetry, the difference of the magnetization dynamics between the two helicities should remain the same when reversing the initial saturation direction. Thus, Figs. 2(a) and 2(b) should be equivalent in absolute value. Yet, this is not the case when comparing the difference of the dynamics between the two helicities in Figs. 2(a) and 2(b). This indicates the presence of a helicity-dependence of the reflectivity. Consequently, to quantitatively determine the $M C D$ from dynamics measurements, it is necessary to remove the HD non-magnetic contributions (see. Supplemental materials [27]).

Fig. 3(a) presents the helicity-independent (HI) ultrafast magnetization change $(\Delta M)$. As previously discussed, $\Delta M$ can also be extracted from a linear combination of the data acquired as defined in Eq. 1 and shown in Figs. 2(a) and 2(b). Fig. 3(a) reveals an ultrafast quenching of the magnetization of about $50 \%$ characterized by a demagnetization time $\tau_{M} \approx 500 \mathrm{fs}$. Then the magnetization slowly relaxes back in a ps-timescale. Notably in Figs. 2(a), 2(b) and 3(a) the error bars are smaller than the symbol size. This HI-magnetization change behavior would be equivalent to pumping the ma-

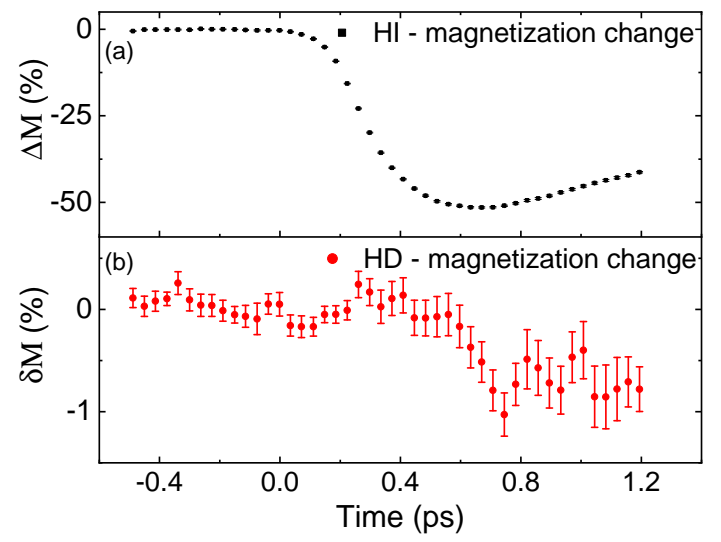

FIG. 3. (a) Helicity-independent $(\Delta M)$ and (b) helicitydependent $(\delta M)$ magnetization change extracted from ultrafast magnetization dynamics induced by circularly polarized light. $\delta M$ is proportional to the magnetic circular dichroism.

terial with linear polarization. Similar results were previously reported for several CoTb alloys [26].

The difference between the two magnetization dynamics arising from the MCD is represented by $\delta M$. This helicity-dependent (HD) magnetization change derived from the TR-MOKE measurements after subtracting the non-magnetic effects is shown in Fig. 3(b). First, the light helicity has no impact on the characteristic demagnetization time. Indeed the quenching of the magnetization occurs in a similar timescale as observed in Figs. 2(a) and 2(b). On the other hand, we report a clear variation of the demagnetization rate of about $0.8 \%-1 \%$ as seen in Fig. 3(b). Importantly, in our femtosecond laser system we measured a fluence fluctuation of less than $0.5 \%$. Hence, we exhibit a $M C D$ in the order of $\lesssim 1 \%$ in a CoTb ferrimagnetic alloy that demonstrates multishot AO-HDS. So far, the MCD was experimentally investigated only in GdFeCo alloys that show single-pulse switching [28] and in a Co/Pt trilayer [23] to explain the AO-HDS. Moreover, Fig. 3(b) unveils that $\delta M<0$, thus the $M C D$ is negative. These findings implies that when pumping with $\sigma^{-}$polarization (resp. $\sigma^{+}$) more light is absorbed by the magnetic medium for the magnetization pointing down (resp. up) leading to a higher demagnetization efficiency.

In order to fully understand the consequence of the MCD on the mechanism of AOS, it is crucial to perform a comparison with AO-HDS experiments as well as helicity-dependent domain wall motion as it was demonstrated in some cases that the laser-induced switching involves domain wall propagation [15, 17, 18]. For this purpose, we used the same linear polarizer and quarter wave plate that allowed us to generate circular polarization in the pump-probe setup. The thin film was saturated with the electromagnet used in the TR-MOKE measurements. This enabled us to avoid any error for 
the light handedness and magnetization direction. The results are displayed in Fig. 4. In Fig. 4(a) and 4(b), a circularly-polarized laser beam is swept over the sample surface at $40 \mu \mathrm{m} \mathrm{s}^{-1}$ with a fluence set to $10 \mathrm{~mJ} \mathrm{~cm} \mathrm{~cm}^{-1}$ for a magnetization pointing up and down, respectively. We see that $\sigma^{-}$(resp. $\sigma^{+}$) polarization reverses the magnetization when the sample is initially saturated in the down (resp. up) direction. In light of the previous measurements of the $M C D$, this means that laser-induced magnetization switching occurs when the magnetization is initially in the most absorbent state according to the light helicity. Thus, AO-HDS ends up in the least absorbent magnetization configuration which was hypothesized in the thermal models describing this phenomenon $[16,21]$.

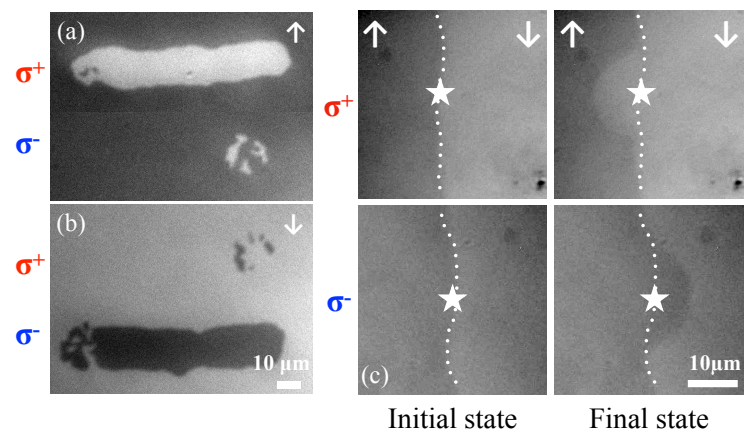

FIG. 4. (a) and (b) Magneto-optical Kerr (MOKE) images taken after sweeping circularly polarized fs laser beam over the CoTb surface for a magnetization initially saturated up $(\uparrow)$ or down $(\downarrow)$. (c) MOKE images taken before and after exposure to a static laser beam centered (white star) on the domain wall and with a fluence below the switching threshold.

Moreover, in Fig. 4(c) we present the results of the helicity-dependent domain wall (HD-DW) motion measurements. Details about the experimental setup can be found in Ref. [18]. The left (resp. right) column indicates the initial (resp. final) domain wall configuration before (resp. after) femtosecond laser irradiation. The laser beam was centered on the DW and the fluence was set below the switching threshold to avoid domain nucleation. We report that $\sigma^{+}$(resp. $\sigma^{-}$) polarization induces an expansion of the magnetization-down (resp. -up) domain. Hence, the direction of the domain wall propagation depends on the light helicity. So far, HD all-optical DW motion was reported only in $\mathrm{Co} / \mathrm{Pt}$ multilayers $[17,18]$. Here, we demonstrated that CoTb alloy that shows multishot AO-HDS [15] also exhibits HD-DW propagation, which provides further evidence of the important role of DW motion in the reversal mechanism in AOS. Besides considering a centered circularly-polarized laser beam, the MCD will lead to an absorption (hence temperature) difference between the two magnetic domains, thus creating a temperature gradient across the DW. By comparing the results of the MCD with the DW motion experiments [Fig. 3(b) and Fig. 4(c)], we can say that the DW moves towards the hotter regions. Therefore, the direction of the laser-induced DW propagation is directly related to the direction of the temperature gradient. As it was proved that multishot AO-HDS involves a HI-domain nucleation followed by a HD-DW motion $[15,17,18]$, it results that thermal effects, e.g. the temperature gradient arising from the MCD, may govern the reversal process in AOS.
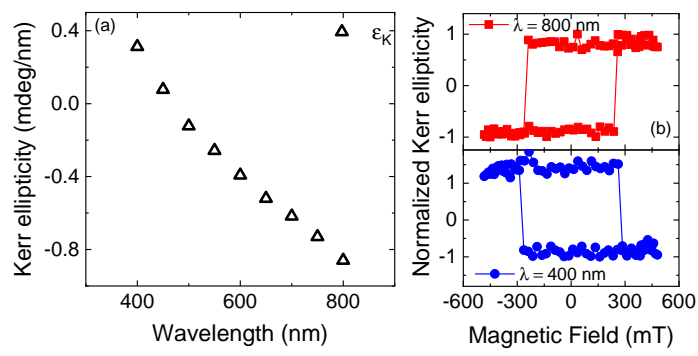

FIG. 5. (a) Magneto-optical Kerr ellipticity spectroscopy measurements. (b) MOKE hysteresis loops obtained with 400 $\mathrm{nm}$ and $800 \mathrm{~nm}$ light.

In order to further investigate the role of MCD and thermal processes in AOS, we carried out spectroscopic Kerr ellipticity measurements. Indeed, when circularlypolarized light is reflected onto an anisotropic magnetic medium, the light electric field's direction experiences a rotation (Kerr rotation) but also a change of the amplitude of its $\mathrm{x}$ - and $\mathrm{y}$ - components (Kerr ellipticity) due to the circular dichroism. Therefore, the sign of the Kerr ellipticity $\left(\varepsilon_{K}\right)$ gives information about the difference of absorption between left- and right- circular polarization. To measure $\varepsilon_{\mathrm{K}}$, we used a custom-made magneto-optical spectrometer based on a photoelastic modulator. More details about this experimental technique can be found in Ref. [29]. The Kerr ellipticity is plotted against the light wavelength in Fig. 5(a). The most striking feature is that the sign of $\varepsilon_{\mathrm{K}}$ changes at $450 \mathrm{~nm}$ as seen also in the MOKE hysteresis loops obtained at $400 \mathrm{~nm}$ and $800 \mathrm{~nm}$ (see Fig. 5(b)). This result indicates that the difference of absorption between left- and right- circular polarization, i.e. the $M C D$, is wavelength dependent and reverses at $450 \mathrm{~nm}$. Therefore, the direction of the temperature gradient across the DW in the previously discussed experiment would change. It is thus expected that the DW would move in the opposite direction than in Fig. 4(c).

In conclusion, in this study we were able to resolve the role of the MCD in AOS by extracting its sign from ultrafast magnetization dynamics in a CoTb alloy that exhibits AO-HDS. Removal of the HI and HD non-magnetic contributions in the TR-MOKE measurements is crucial to accurately determine the $M C D$. We found out that AOS occurs when the magnetization is initially in the most absorbent state according to the light helicity. 
Moreover, we proved that a ferrimagnetic alloy can also exhibit laser-induced DW motion. This would tend to confirm a pure thermal mechanism based on the MCD that can be described in two steps. First, a multi-domain state is formed by thermal demagnetization. Then, due to the temperature gradient across DWs arising from the MCD, some domains will shrink or grow depending on the magnetization direction and light helicity. Finally, our experimental results validate the assumptions made in the thermal models of multishot AO-HDS [16, 21].

This work was supported partly by the French PIA project "Lorraine Université d'Excellence", reference ANR-15-IDEX-04-LUE.

* yassine.quessab@nyu.edu

$\dagger$ gregory.malinowski@univ-lorraine.fr

[1] C. D. Stanciu, F. Hansteen, A. V. Kimel, A. Kirilyuk, A. Tsukamoto, A. Itoh, and T. Rasing, Physical Review Letters 99, 047601 (2007).

[2] E. Beaurepaire, J. C. Merle, A. Daunois, and J. Y. Bigot, Physical Review Letters 76, 4250 (1996).

[3] I. Radu, K. Vahaplar, C. Stamm, T. Kachel, N. Pontius, H. A. Dürr, T. A. Ostler, J. Barker, R. F. L. Evans, R. W. Chantrell, A. Tsukamoto, A. Itoh, A. Kirilyuk, T. Rasing, and A. V. Kimel, Nature 472, 205 (2011).

[4] T. A. Ostler, J. Barker, R. F. L. Evans, R. W. Chantrell, U. Atxitia, O. Chubykalo-Fesenko, S. El Moussaoui, L. Le Guyader, E. Mengotti, L. J. Heyderman, F. Nolting, A. Tsukamoto, A. Itoh, D. Afanasiev, B. A. Ivanov, A. M. Kalashnikova, K. Vahaplar, J. Mentink, A. Kirilyuk, T. Rasing, and A. V. Kimel, Nature Communications 3, 666 EP (2012).

[5] J. Gorchon, R. B. Wilson, Y. Yang, A. Pattabi, J. Y. Chen, L. He, J. P. Wang, M. Li, and J. Bokor, Physical Review B 94, 184406 (2016).

[6] J. P. van der Ziel, P. S. Pershan, and L. D. Malmstrom, Physical Review Letters 15, 190 (1965).

[7] M. Battiato, G. Barbalinardo, and P. M. Oppeneer, Physical Review B 89, 014413 (2014).

[8] A. V. Kimel, A. Kirilyuk, P. A. Usachev, R. V. Pisarev, A. M. Balbashov, and T. Rasing, Nature 435, 655 (2005).

[9] G.-M. Choi, A. Schleife, and D. G. Cahill, Nature Communications 8, $15085 \mathrm{EP}$ (2017).

[10] S. Mangin, M. Gottwald, C.-H. Lambert, D. Steil, V. Uhlír, L. Pang, M. Hehn, S. Alebrand, M. Cinchetti, G. Malinowski, Y. Fainman, M. Aeschlimann, and E. E. Fullerton, Nature Materials 13, 286 (2014).

[11] C.-H. Lambert, S. Mangin, B. S. D. C. S. Varaprasad, Y. K. Takahashi, M. Hehn, M. Cinchetti, G. Malinowski,
K. Hono, Y. Fainman, M. Aeschlimann, and E. E. Fullerton, Science 345, 1337 (2014).

[12] X. Yong, M. Deb, G. Malinowski, M. Hehn, W. Zhao, and S. Mangin, Advanced Materials 29, 1703474 (2017).

[13] S. Alebrand, M. Gottwald, M. Hehn, D. Steil, M. Cinchetti, D. Lacour, E. E. Fullerton, M. Aeschlimann, and S. Mangin, Applied Physics Letters 101, 162408 (2012).

[14] M. S. El Hadri, P. Pirro, C. H. Lambert, N. Bergeard, S. Petit-Watelot, M. Hehn, G. Malinowski, F. Montaigne, Y. Quessab, R. Medapalli, E. E. Fullerton, and S. Mangin, Applied Physics Letters 108, 092405 (2016).

[15] M. S. El Hadri, P. Pirro, C. H. Lambert, S. Petit-Watelot, Y. Quessab, M. Hehn, F. Montaigne, G. Malinowski, and S. Mangin, Physical Review B 94, 064412 (2016).

[16] J. Gorchon, Y. Yang, and J. Bokor, Physical Review B 94, 020409 (2016).

[17] R. Medapalli, D. Afanasiev, D. K. Kim, Y. Quessab, S. Manna, S. A. Montoya, A. Kirilyuk, T. Rasing, A. V. Kimel, and E. E. Fullerton, Physical Review B 96, 224421 (2017).

[18] Y. Quessab, R. Medapalli, M. S. El Hadri, M. Hehn, G. Malinowski, E. E. Fullerton, and S. Mangin, Physical Review B 97, 054419 (2018).

[19] T. D. Cornelissen, R. Córdoba, and B. Koopmans, Applied Physics Letters 108, 142405 (2016).

[20] P. Nieves and O. Chubykalo-Fesenko, Physical Review Applied 5, 014006 (2016).

[21] M. O. A. Ellis, E. E. Fullerton, and R. W. Chantrell, Scientific Reports 6, 30522 (2016).

[22] R. John, M. Berritta, D. Hinzke, C. Müller, T. Santos, H. Ulrichs, P. Nieves, J. Walowski, R. Mondal, O. Chubykalo-Fesenko, J. McCord, P. M. Oppeneer, U. Nowak, and M. Münzenberg, Scientific Reports 7, 4114 (2017).

[23] Y. Tsema, G. Kichin, O. Hellwig, V. Mehta, A. V. Kimel, A. Kirilyuk, and T. Rasing, Applied Physics Letters 109, 072405 (2016).

[24] M. S. El Hadri, M. Hehn, P. Pirro, C.-H. Lambert, G. Malinowski, E. E. Fullerton, and S. Mangin, Physical Review B 94, 064419 (2016).

[25] B. Koopmans, G. Malinowski, F. Dalla Longa, D. Steiauf, M. Fähnle, T. Roth, M. Cinchetti, and M. Aeschlimann, Nature Materials 9, 259 (2010).

[26] S. Alebrand, U. Bierbrauer, M. Hehn, M. Gottwald, O. Schmitt, D. Steil, E. E. Fullerton, S. Mangin, M. Cinchetti, and M. Aeschlimann, Physical Review B 89, 144404 (2014).

[27] "See supplemental materials at for details about the helicity-dependent ultrafast magnetization dynamics equation and derivation of the mcd,".

[28] A. R. Khorsand, M. Savoini, A. Kirilyuk, A. V. Kimel, A. Tsukamoto, A. Itoh, and T. Rasing, Physical Review Letters 108, 127205 (2012).

[29] M. Deb, M. Vomir, J.-L. Rehspringer, and J.-Y. Bigot, Applied Physics Letters 107, 252404 (2015). 